\title{
ALGEBRAS OVER INFINITE FIELDS
}

\author{
A. S. AMITSUR
}

1. Introduction. Let $A$ be an algebra over a field $F$. We shall be mainly interested in infinite-dimensional algebras over infinite fields $F$. Actually most of the results will be obtained for nondenumerable fields $F$.

The present paper starts with studying the spectrum of the elements of $A$. More precisely, the complement of the spectrum in $F$ is studied. It seems that from an algebraic point of view the complement is of greater interest than the spectrum itself. As a consequence of the study of the complement it is shown that the Jacobson radical [2] of algebras $A$ for which $(A: F)<$ the cardinal number of $F$ is always the maximal nil ideal of $A$. Furthermore, for algebras satisfying this inequality, the fact that an element is algebraic or nil can be expressed by conditions on the complement of the spectrum of that element.

These results are then applied to provide an affirmative solution for some well known structure problems of rings, though only for algebras over nondenumerable fields. The first problem is the following problem of Koethe [6]: "Does the maximal nil ideal of $A$ contain all one-sided nil ideals of $A$ ?" The others are two problems proposed by Jacobson [3]: "Let $A$ be an algebraic algebra over $F$. Does it follow that the matrix ring $A_{n}$ over $A$ is also algebraic and is the extension algebra $A_{H}$ algebraic over $H$ for any extension $H$ of $F$ ?"

These problems are related to the problem of Kurosh [7] and Levitzki [9]: "Is every algebraic algebra locally finite?" ${ }^{\text {It was }}$ pointed out by Levitzki [9] that an affirmative solution for this problem will provide an affirmative solution for the problem of Koethe, and it was observed by Jacobson that this will imply also an affirmative answer for the other structure problems of algebraic algebras. In the present paper we attack the problems of Koethe and Jacobson avoiding the problem of Kurosh which still remains unsolved even in the case of nondenumerable fields $F$, for which we provide an affirmative answer for the other problems.

An important tool in attacking the problem of Kurosh are the two radicals defined by Levitzki: the semi-nilpotent radical [8] and the locally finite kernel [10]. The cases of the known affirmative solution

Received by the editors February 5, 1955.

1 The special case of this problem for nil rings was proposed independently by Levitzki in [9] in connection with the problem of Koethe. 
for the problem of Kurosh (e.g. rings with identities [5]) are readily obtained by the use of these radicals $[4 ; 10]$. In the present paper we define two new radicals containing, respectively, the semi-nilpotent radical and the locally finite kernel. An algebra $A$ is called an LBI (LBD) algebra if every finitely generated submodule of $A$ contains nilpotent (algebraic) elements of bounded index (degree). It is an open question whether these properties are really new properties or whether they are equivalent to semi-nilpotency and to local finiteness, respectively. Nevertheless, the study of the LBI radical and the LBD radical can be carried out and it is shown that these radicals satisfy all required properties of radicals [1]. It is proved that the $\mathrm{LBI}$ radical coincides with the maximal nil ideal of an algebra and the LBD radical is the maximal algebraic ideal of an algebra over nondenumerable fields. These results are applied to give an affirmative answer to the problems of Koethe and Jacobson for such algebras.

2. The Jacobson radical. Let $A$ be an algebra over a field $F$. We recall that an element $a \in A$ is said to be a quasi-regular element in $A$ if there exists an element $b \in A$ satisfying $a+b+a b=0$. The element $b$ is called a quasi-inverse of $a$. Generally, the quasi-inverse is not uniquely determined, but if $b$ and $c$ are two quasi-inverses of $a$ then their difference $x=b-c$ satisfies: $x+x a=0$.

By the spectrum $S(a)=S_{F}(a)$ of an element $a \in A$ we mean the set of all $\lambda \in F$ for which the element $-\lambda^{-1} a$ is not quasi-regular. It is accepted to include 0 in $S(a)$ unless $A$ contains a unit and $a$ is regular. By $C(a)=C_{F}(a)$ we shall denote the complement of the spectrum $S(a)$ in $F$. That is, $C(a)$ is the set of all $\lambda \in F$ for which $-\lambda^{-1} a$ is quasi-regular. We shall also denote by $N(a)=N_{F}(a)$ the set of all $\lambda \in F$ for which the equation $(\lambda-a) x=0$ has a nonzero solution in $A$.

Lemma 1. If $a \in A$ is algebraic then $N(a)=S(a)$ and the union $N(a) \cup C(a)=F$.

Let $f(X)=0$ be the minimal equation of $a$ over $F$ and let $\lambda_{1}, \cdots, \lambda_{r}$ be the roots of this equation which lie in $F$; then since $f(X)$ $=\left(\lambda_{i}-X\right) g_{i}(X)$ it follows that $0=f(a)=\left(\lambda_{i}-a\right) g_{i}(a)$ and $g_{i}(a) \in A .^{2}$ Thus $\lambda_{i} \in N(a)$. If $\lambda \neq \lambda_{i}, f(\lambda) \neq 0$ and let

$$
g(X)=\left(X-\lambda f(\lambda)^{-1} f(X)\right) /(X-\lambda)
$$

then $g(X)$ is a polynomial in $X$ and one readily verifies that ${ }^{2} g(a)$ $\in A$ and $g(a)$ is the quasi-inverse of $-\lambda^{-1} a$. The rest is readily verified.

2 No unit has to be assumed to exist in $A$. 
REMARK 1. Furthermore, one readily observes that in this case $N(a)$ is finite and the quasi-inverses of $-\lambda^{-1} a$ commute with $a$ since they belong to the algebra generated by $a$. Here $N(a)$ and $C(a)$ are disjoint sets, which is not necessarily true in the general case.

Denote by $a_{\lambda}$ a quasi-inverse of $-\lambda^{-1} a$ for $\lambda \in C(a)$.

Theorem 1. If $a \in A$ is not algebraic over $F$ and $\left\{\lambda_{j}\right\}$ is a set of nonzero different elements of $C(a)$, then the corresponding set $\left\{a_{\lambda_{j}}\right\}$ are linearly independent over $F$.

Since $a_{\lambda}$ is a quasi-inverse of $-\lambda^{-1} a$ it follows that $-\lambda^{-1} a+a_{\lambda}$ $-\lambda^{-1} a a_{\lambda}=0$. Hence:

$$
a-\lambda a_{\lambda}+a a_{\lambda}=0 .
$$

This implies that

$$
\lambda a_{\lambda}=a+a a_{\lambda}
$$

Multiplying (1) by $\lambda$ and substituting (2) one obtains

$$
0=\lambda a-\lambda^{2} a_{\lambda}+a \lambda a_{\lambda}=\lambda a-\lambda^{2} a_{\lambda}+a\left(a+a a_{\lambda}\right) .
$$

Hence

$$
\lambda a+a^{2}-\lambda^{2} a_{\lambda}+a^{2} a_{\lambda}=0 .
$$

Multiplying now (1.2) by $\lambda$ and substituting (2) and continuing in this way we obtain:

$$
\lambda^{i-1} a+\lambda^{i-2} a^{2}+\cdots+a^{i}-\lambda^{i} a_{\lambda}+a^{i} a_{\lambda}=0 .
$$

For $i=1$ this formula is exactly (1).

We shall prove now that if the set $\left\{a_{\lambda_{j}}\right\}$ is linearly dependent over $F$ then $a$ is algebraic. Indeed let:

$$
\sum_{j=1}^{r} \alpha_{j} a_{\lambda_{j}}=0, \alpha_{j} \in F \text { and not all } \alpha_{j}=0 .
$$

Put $\lambda=\lambda_{j}$ in (1.i); multiply it by $\alpha_{j}$ and sum over all $j$. This yields:

$$
\begin{aligned}
& f_{i}(a)-\sum_{j} \lambda_{j}^{i} \alpha_{j} a_{\lambda_{j}}+a^{i} \sum_{j} \alpha_{j} a_{\lambda_{j}}=0 \text { where } \\
& f_{i}(a)=a \sum_{j} \alpha_{j} \lambda_{j}^{i-1}+a^{2} \sum_{j} \alpha_{j} \lambda_{j}^{i-2}+\cdots+a^{i} \sum \alpha_{j} .
\end{aligned}
$$

This holds for $i=1,2, \cdots$. Setting $f_{0}(a)=0$, this together with (3) implies 


$$
f_{i}(a)=\sum_{j=1}^{r} \lambda_{j}^{i} \alpha_{j} a_{\lambda_{j}} \quad \text { for } i=0,1, \cdots, r-1 .
$$

Write (4) in a matrix form:

$$
\left(f_{0}(a) \cdots f_{r-1}(a)\right)=\left(\alpha_{\lambda_{1}} a_{\lambda_{1}} \cdots \alpha_{r} a_{\lambda_{r}}\right)\left(\lambda_{j}^{i}\right) .
$$

Since all the $\lambda_{j}$ were assumed to be different the matrix $\left(\lambda_{j}^{1}\right)$ is regular in $F$, hence multiplying $\left(4^{\prime}\right)$ on the left by the matrix $\left(\lambda_{j}^{i}\right)^{-1}$ we obtain that $\alpha_{j} a_{\lambda j}=p_{j}(a)$ for some polynomial $p_{j}(a)$ of $a$. Since not all the $\alpha_{j}$ are zero it follows that $a_{\lambda}=q(a)$ for some $\lambda$ and some polynomial $q(a)$ of $a$. Thus (1) implies that $Q(a)=a-\lambda q(a)+a q(a)=0$. Clearly $\lambda \neq 0$ implies that $q(a) \neq 1$. Hence, $Q(X)$ is a nonzero polynomial and this proves that $a$ is algebraic.

Note that if $q(X)$ is a polynomial of degree $t$ then $Q(X)$ is of degree $t+1$. From $\left(4^{\prime}\right)$ one readily observes that $p_{j}(X)$ and, therefore, also $q(X)$ are at most of degree $r-1$. Hence the previous proof yields for algebraic elements that:

Corollary 1. If $a \in A$ is algebraic of degree $s$ and $\lambda_{1}, \cdots, \lambda_{r}$, $r \leqq s-1$ are nonzero elements of $C(a)$, then $a_{\lambda_{1}}, \cdots, a_{\lambda_{r}}$ are linearly independent over $F$.

In view of Remark 1 and the preceding theorem it follows that:

THEOREM 2. If $F$ is an infinite field, then the set $\left\{a_{\lambda}\right\}$ where $\lambda$ ranges over all the elements of $C(a)$ is dependent over $F$ if and only if $a$ is algebraic over $F$.

An immediate consequence of this theorem is:

COROLlaRY 2. If a is not algebraic then the cardinal number of the set $C(a)$ is $\leqq(A: F)$.

Clearly, $C(a)=F$ for every element $a$ of a division algebra $A$. Hence:

COROllary $2^{\prime}$. If $A$ is not an algebraic division algebra, then the cardinal number of the base field $F$ is $\leqq(A: F)$.

Lemma 2. For $\lambda \in N(a)$ denote by $x_{\lambda}$, a nonzero solution of the equation $(\lambda-a) x=0$; then the set $\left\{x_{\lambda}\right\}$ for all $\lambda \in N(a)$ is linearly independent over $F$.

Indeed let $\left\{x_{\lambda j}\right\}$ be a maximal set of linearly independent elements taken from the set of all $\left\{x_{\lambda}\right\}$. If the two sets do not coincide then $x_{\lambda}=\sum \alpha_{j} x_{\lambda j}$ for some $\lambda \in N(a)$ and $\lambda \neq \lambda_{j}$ for all $j$. Multiplying this equality on the left by $a$ we obtain, in view of the definition of $N(a)$, 
that $\lambda x_{\lambda}=\sum \alpha_{j} \lambda_{j} x_{\lambda_{j}}$. It follows now by the fact that the $x_{\lambda_{j}}$ are independent over $F$ that $\lambda \alpha_{j}=\alpha_{j} \lambda_{j}$ for all $j$. Since $x_{\lambda} \neq 0$, at least one of the $\alpha_{j} \neq 0$; hence $\lambda=\lambda_{j}$ for some $j$. Contradiction.

Notations. For any given set $S$ we denote by card $S$ the cardinal number of the set $S$. We shall denote by $A_{H}$ the algebra obtained from $A$ by extending the base field to $H$. Namely, $A_{H}=A \otimes H$.

We shall deal with two radicals of the algebra $A$ : the Jacobson radical of $A[2]$ which we shall denote by $\Im(A)$ and the upper radical of $A$, denoted by $\Omega(A)$. The latter is defined as the maximal nil ideal of $A$. In case $\Re(A)$ contains also all one-sided nil ideals of $A$, this radical is known as the Koethe radical [6] of $A$.

In the following results we deal with algebras $A$ over $F$ for which card $F>(A: F)$. For these algebras the condition that an element is algebraic or nil can be expressed by conditions on the set $C(a)$ or on $S(a)$ :

Theorem 3. If $F$ is infinite and card $F>(A: F)$ then an element $a \in A$ is algebraic over $F$ if and only if card $F=\operatorname{card} C(a)$. And in this case $S(a)$ contains only a finite number of elements.

If $F$ is algebraically closed and satisfies the preceding condition then $a \in A$ is nilpotent if and only if $S(a)$ contains only the zero.

The sufficiency of the two parts of the proof follows immediately by the proof of Lemma 1. The necessity of the first part is an immediate consequence of Corollary 2 and the rest of the first part follows now by the proof of Lemma 1. Furthermore, that proof shows that $S(a)$ contains the set of roots of the minimal equation of $a$. Hence if $F$ is algebraically closed and $S(a)=(0)$ it follows that card $C(a)=\operatorname{card} F$ and, therefore, $a$ is algebraic. Consequently, the minimal equation of $a$ is of the form $X^{n}=0$, i.e., $a$ is nilpotent.

This theorem fails if card $F \leqq(A: F)$. Since, for elements belonging to the Jacobson radical of an algebra $A$, the spectrum can contain at most the zero but yet one can construct examples of algebras over any field $F$ whose radical $\Im(A)$ does not contain algebraic elements.

The preceding theorem can be applied to prove the converse of Lemma 1.

Lemma 3. If card $F>(A: F)$ then an element $a \in A$ is algebraic over an infinite field $F$ if and only if $N(a) \cup C(a)=F$.

Indeed, it follows by Lemma 2 that card $N(a) \leqq(A: F)$; hence if $N(a) \cup C(a)=F$ we obtain that card $C(a)=$ card $F$, since card $N(a)$ $\leqq(A: F)<$ card $F$. This implies by the preceding theorem that $a$ is algebraic. The converse is stated in Lemma 1.

We are now in position to show: 
TheOREM 4. If card $F>(A: F)$ then the quasi-regular one-sided ideals of $A$ are its nil one-sided ideals.

It is known that nil ideals are quasi-regular ideals [2]. To prove the converse we consider a right quasi-regular ideal $J$ in $A$. Let $a \in J$; then $-\lambda^{-1} a x \in J$ is quasi-regular for fixed $x \in A$ and for every $\lambda \in F$. This readily implies that card $C(a x)=$ card $F$. It follows, therefore, by Theorem 3 that $a x$ is algebraic. Thus, the right ideal $a A$ is an algebraic ideal in $A$. Since $a A \subset J$ it follows that $a A$ is also quasiregular, but it is known [2] that algebraic quasi-regular ideals are nil ideals. In particular, $a^{2} \in a A$ is nilpotent and consequently $a$ is also nilpotent. This proves that $J$ is a right nil ideal.

This immediately implies:

Corollary 3. If card $F>(A: F)$ then $\Im(A)=\Re(A)$.

Indeed, since $\Im(A)$ is quasi-regular it is a nil ideal by the preceding theorem; hence $\Im(A) \subseteq \Re(A)$. The converse $\Omega(A) \subseteq \Im(A)$ is well known. Hence $\Omega(A)=\Im(A)$.

The preceding corollary can be applied to the following case: One easily verifies that a finitely generated algebra over $F$ has at most a countable base, hence the preceding corollary implies that:

COROLLARY 4. The Jacobson radical of a finitely generated algebra over a nondenumerable field is its nil radical.

Furthermore, the preceding theorem yields an affirmative answer to the problem of Koethe in the case of algebras of relatively low dimensionality. That is:

CoROllary 5. If card $F>(A: F)$ then the union of right nil ideals in $A$ is also a nil right ideal contained in $\Re(A)$; and every right nil ideal is contained in a two-sided nil ideal of $A$.

This is an immediate consequence of Theorem 4 and the fact that this corollary holds for quasi-regular right ideals [2].

This corollary will be later extended for arbitrary algebras over nondenumerable fields.

3. Algebras over nondenumerable fields. In the present section we confine ourselves to nondenumerable fields $F$.

Let $V^{r}$ be an $r$-dimensional vector space over $F$. A subset $U \subset V^{r}$ is an algebraic set if $U$ is the totality of the points $\left(x_{1}, \cdots, x_{r}\right) \in V^{r}$ which satisfy a (finite or infinite) set of polynomial equations $f_{i}\left(X_{1}, \cdots, X_{r}\right)=0$. The polynomials $f_{i}(X)$ will be said to define the algebraic set $U$. 
The important property of the nondenumerable fields for our purpose is:

Lemma 4. Let $U_{1}, U_{2}, U_{3}, \cdots$ be a denumerable set of algebraic sets of $V^{r}$; then either $U U_{i} \neq V^{r}$ or else $U_{n}=V^{r}$ for some integer $n$.

The proof is carried out by induction on the dimension $r$ of the space $V^{r}$. For $r=1$, the sets $U_{i}$ are defined by polynomials in one variable; hence if these polynomials are not identically zero the algebraic set $U_{i}$ contains only a finite number of points. Thus if $U_{i} \neq V^{\prime}$ for all $i$ then each of them is finite and, therefore, their union $U U_{i}$ is denumerable but $V^{1}$ which in this case coincides with $F$ is, by assumption, nondenumerable. Consequently, $U U_{i} \neq V^{1}$.

This part of the proof fails for denumerable fields $F$. Since clearly every finite subset of $V^{1}$ is an algebraic set, by suitably choosing the sets $U_{i}$ one can obtain a denumerable set of algebraic sets for which $U U_{i}$ will be the whole denumerable space $V^{1}$.

Let $r>1$. Let $f_{i j}\left(X_{1}, \cdots, X_{r}\right)=\sum_{k} f_{i j k}\left(X_{1}, \cdots, X_{r-1}\right) X_{r}^{k}$ be the defining polynomials of the algebraic set $U_{i}$. Let $W_{i}$ be the algebraic subset of $V^{r-1}$ defined by the algebraic equations: $f_{i j k}\left(X_{1}, \cdots, X_{r-1}\right)$ $=0$ for fixed $i$ and all possible $j$ and $k$. Namely

$$
\begin{gathered}
W_{i}=\left\{\left(\alpha_{1}, \cdots, \alpha_{r-1}\right) \in V^{r-1} \text { for which }\left(\alpha_{1}, \cdots, \alpha_{r-1}, x_{r}\right)\right. \\
\left.\in U_{i} \text { for all } x_{r} \in F\right\} .
\end{gathered}
$$

It follows now easily that $W_{i}$ is also a denumerable set of algebraic sets in $V^{r-1}$. Hènce, by induction, either $W_{n}=V^{r-1}$ or $U W_{i} \neq V^{r-1}$. In the first case it follows that $f_{i j k}\left(X_{1}, \cdots, X_{r-1}\right) \equiv 0$ and consequently also $f_{i j}(X) \equiv 0$. This proves that $U_{m}=V^{r-1}$ which proves the lemma. If the second case holds, then there exists at least one point $\left(\alpha_{1}, \cdots\right.$, $\left.\alpha_{r-1}\right) \in V^{r-1}$ which does not belong to the union $U W_{i}$. Thus this point satisfies the property that for each $i$ there exists at least one $j$ and $k$ such that $f_{i j k}\left(\alpha_{1}, \cdots, \alpha_{r-1}\right) \neq 0$. Consequently, at least one of the polynomials $f_{i j}\left(\alpha_{1}, \cdots, \alpha_{r-1}, X_{r}\right) \neq 0$. Let $W_{i}^{\prime}$ be the set of all $x_{r} \in F$ which satisfies all the equations $f_{i j}\left(\alpha_{1}, \cdots, \alpha_{r-1}, x_{r}\right)=0$ for all $j$. Since not all these polynomials are identically zero, this set is finite. Hence $U W_{i}^{\prime}$ is denumerable. There exists, therefore, an element $\alpha \in F$ such that $\alpha \notin \cup W_{i}^{\prime}$. This means that for each $i$ there exists at least one $j$ so that $f_{i j}\left(\alpha_{1}, \cdots, \alpha_{r-1}, \alpha\right) \neq 0$. It follows, therefore, that $\left(\alpha_{1}, \cdots, \alpha_{r-1}^{\prime}, \alpha\right) \notin U_{i}$ for all $i$. This completes the proof of the lemma.

This lemma will be used later.

Lemma 5. If $F$ is any field and $a_{1}, \cdots, a_{r}$ is a finite set of elements 
in $A$, then the set of all $\left(x_{1}, \cdots, x_{r}\right) \in V^{r}$ for which the element $x_{1} a_{1}$ $+\cdots+x_{r} a_{r}$ is algebraic (nilpotent) of degree (index) $\leqq n$ is an algebraic set $U_{n}\left(W_{n}\right)$ in $V^{r}$.

Choose a basis $\left\{e_{\nu}\right\}$ of $A$ over $F$ and let $a_{i}=\sum \alpha_{i \nu} e_{\nu}$. Let $e_{\nu} e_{\mu}$ - $\sum \gamma_{\nu \mu \rho} e_{\rho}$; then one readily verifies that $\left(\sum x_{i} a_{i}\right)^{n}=\sum p_{n \nu}(x) e_{\nu}$ where $p_{n \nu}(x)=p_{n \nu}\left(x_{1}, \cdots, x_{r}\right)$ is a form of degree $\leqq n$ in the $x_{i}$. This readily implies that the algebraic set $W_{n}$ defined by the set of all equations $p_{n \nu}(X)=0$ satisfies the corresponding requirement of the lemma.

The condition that $\sum x_{i} a_{i}$ is algebraic of degree $\leqq n$ is equivalent to the existence of a set of elements $\lambda_{0}, \cdots, \lambda_{n}$ in $F$ not all zero satisfying $\sum_{\rho=0}^{n} \lambda_{\rho}\left(\sum x_{i} a_{i}\right)^{\rho}=\sum_{\nu}\left(\sum_{\rho} \lambda_{\rho} p_{\rho \nu}(x)\right) e_{\nu}=0 .^{3}$ Equivalently,

$$
\lambda_{0} p_{0 \nu}(x)+\lambda_{1} p_{1 \nu}(x)+\cdots+\lambda_{n} p_{n \nu}(x)=0 \text { for all } \nu \text {. }
$$

Consider (5) as a set of infinite homogeneous linear equations in the $n+1$ unknown $\lambda_{0}, \cdots, \lambda_{n}$; then a necessary and sufficient condition for the existence of a nonzero solution is that all the determinants of $\operatorname{order}^{4}(n+1)^{2}$ formed by the coefficients of $(5)$ are zero. Let $P(x ; \nu)$ $=\left|p_{0 v_{0}}(x), \cdots, p_{n v_{n}}(x)\right|$ be the determinant formed by the $\nu_{0}, \cdots, \nu_{n}$ rows of the coefficients of the equations (5). Thus the preceding arguments show that the algebraic set $U_{n}$ defined by the set of the polynomial equations $P(X ; \nu)=0$ where $(\nu)=\left(\nu_{0}, \cdots, \nu_{n}\right)$ ranges over all possible choices of rows satisfies the requirement of our lemma.

Note that the polynomial $p_{n v}(X)$ is a form of degree $\leqq n$ and the polynomial $P(X ; \nu)$ is a form of degree $\leqq 0+1+\cdots+n=n(n+1) / 2$. Consider now the case of two elements $a, b \in A$. Then a necessary and sufficient condition that $a+x b$ is algebraic (nilpotent) of degree (index) $\leqq n$ is that $P(1, x ; \nu)=0\left(p_{n \nu}(1, x)=0\right)$ for all $(\nu)$. Hence if these polynomials are not identically zero, it follows by their degrees that the number of solutions of these equations is at most $n(n+1) / 2$ $(n)$. This proves:

COROLlaRY 6. Let $F$ be an arbitrary field and $A$ an algebra over $F$. Let $a, b \in A$. Then either every element of the form $a+x b, x \in F$ is algebraic (nilpotent) of degree (index) $\leqq n$ or at most $n(n+1) / 2(n)$ of these elements are algebraic (nilpotent) of degree (index) $\leqq n$.

The preceding lemmas yield:

Theorem 5. If $A$ is an algebraic (nil) algebra over a nondenumerable

${ }^{3}$ If $A$ does not contain a unit we omit the index $=0$.

- Of order $n^{2}$ if $A$ does not contain a unit. 
field $F$, then every finitely generated submodule of $A$ is of bounded degree (index).

For let $a_{1}, \cdots, a_{r}$ generate a submodule of $A$. Consider the algebraic sets $U_{n}$ and $W_{n}$ defined in the preceding lemma. Since $A$ is algebraic (nil) it follows that every element of the form $\sum x_{i} a_{i}$ is algebraic (nil). In other words this means that $U U_{n}=V^{r}\left(U W_{n}=V^{r}\right)$. It follows now by Lemma 4 that $U_{n}=V^{r}$ for some integer $n\left(W_{n}=V^{r}\right)$ and this implies that every element of the form $\sum x_{i} a_{i}$ is algebraic (nil) of degree $\leqq n$.

Note that the preceding proof made no use of the fact that the whole algebra is algebraic (nil), only that the module generated by the $a_{i}$ has this property. Hence, we actually proved:

COROLlaRY 7. Every finitely generated algebraic (nil) module of an algebra $A$ over a nondenumerable field is of bounded degree (index).

4. The LBD and the LBI radicals. The results of the preceding section lead us to a definition of two new properties of algebras.

Definition. An algebraic algebra $A$ over a field $F$ will be said to be locally of bounded degree if every finitely generated submodule of $A$ consists of elements of bounded degree. Such an algebra will be called an LBD algebra.

A nil algebra will be said to be locally of bounded index if every finitely generated submodule of $A$ is of bounded index. These algebras will be called LBI algebras.

REMARK 2. Clearly Theorem 5 states that every algebraic algebra over a nondenumerable field is an LBD algebra; and every nil algebra over a nondenumerable field is an LBI algebra.

Lemma 6. If $A$ is an LBD (LBI) algebra over an infinite field $F$ and $H \supset F$ is an extension field of $F$, then the extension algebra $A_{H}$ is also an $L B D(L B I)$ algebra.

Let $\bar{a}_{j}=\sum a_{j k} h_{j k}, a_{j k} \in A$ and $h_{j k} \in H$, be a finite set of elements of $A_{H}$. The module generated by the elements $\bar{a}_{j}$ over $H$ is clearly contained in the module generated by the elements $a_{j k}$, which are also finite, over $H$. It suffices, therefore, to show that the finitely generated submodules of $A_{H}$ generated by elements of $A$ are of bounded degree (index).

We deal first with the LBD property. Let $a_{1}, \cdots, a_{r} \in A$ and let $\left\{e_{\nu}\right\}$ be a basis of $A$ over $F$, then $\left\{e_{\nu}\right\}$ is also a basis of $A_{H}$ over $H$. One readily observes that using the same base $\left\{e_{\nu}\right\}$ both for $A$ and for $A_{H}$ in the proof of Lemma 5 , one obtains the same polynomials 
$p_{n \nu}(x)$ and $P(x ; \nu)$. Thus an element $\sum x_{i} a_{i}$ is algebraic of degree $\leqq n$ in $A$ or in $A_{H}$ if and only if $P(x ; \nu)=0$ for all possible $(\nu)$. Now $A$ was assumed to be an LBD algebra, hence $P(x ; \nu)=0$ for all $\left(x_{i}\right)$ for some integer $n$. Since $F$ is infinite it follows that $P(X ; \nu) \equiv 0$. This in turn implies that $P(x ; \nu)=0$ for all $x_{i} \in H$ which proves that the elements of the form $\sum x_{i} a_{i}$ in $A_{H}$ are algebraic of degree $\leqq n$. Thus $A_{H}$ is also an LBD algebra.

A similar argument about the polynomials $p_{n v}(X)$ shows that if $A$ is LBI algebra then $A_{H}$ is also.

We study now the properties LBD and LBI from the point of view developed in [1]. The results of the three parts of [1] will be quoted by the latin numeral of the part quoted followed by the number of the result quoted of that part. We shall restrict ourselves to infinite fields $F$.

Our main aim is to show:

Theorem 6. The properties $L B D$ and $L B I$ are $R Z$ properties in the sense of II, $\$ 6$ and satisfy condition $\left(\mathrm{F}_{r}\right)$ of II, $\$ 2$.

It is easily shown that both LBD and LBI satisfy the conditions (A), (B), $\left(\mathrm{C}_{2}\right)$ and $\left(\mathrm{D}_{r}\right)$ (which included (D)) of II ( $\$ 2$, p. 104), and also condition $(Z)$ of II, $\$ 6$. The proof of $\left(C_{1}\right)$ of II for the two properties is similar and we restrict ourselves to the LBD property. To prove $\left(\mathrm{C}_{1}\right)$ we have to show that if $A$ is an algebra containing an ideal $Q$ such that both $Q$ and $\bar{A}=A / Q$ are LBD algebras, then $A$ is also. To this end we consider a field $H \supset F$ such that $H$ is nondenumerable. One readily verifies that $Q_{H}=Q \otimes H$ is an ideal in $A_{H}=A \otimes H$ and that $\bar{A}_{H}=\bar{A} \otimes H \cong A_{H} / Q_{H}$. Since $(A: F)=\left(A_{H}: H\right)$ it follows that the results of the preceding sections hold for the algebra $A_{H}$. By Lemma 6 it follows that $Q_{H}$ and $\bar{A}_{H}$ are algebraic; hence clearly $A_{H}$ is algebraic. This, in view of Remark 2, yields that $A_{H}$ and, therefore, also $A$ are LBD algebras. The last fact follows since the degrees of elements of $A$ do not change when passing to $A_{H}$.

Condition $\left(\mathrm{F}_{r}\right)$ of II, $\$ 2$ states, in our case, that semi-simple LBD (LBI) algebras do not contain one-sided LBD (LBI) algebras, which in view of II, Lemma 3.1, is equivalent to the fact that if $J$ is a right LBD (LBI) ideal in an algebra $A$ then the ideal $A J$ has the same property. To prove this, consider a field $H \supset F$ such that card $H$ $>(A: F)$ and hence card $H>\left(A_{H}: H\right)$. Consider the right ideal $J_{H}=J \otimes H$ in $A_{H} . J_{H}$ is again an LBD (LBI) algebra in $A_{H}$. The proof now differs for the two properties LBD and LBI and we first dispense with the LBI property. For this property it follows by Theorem 4, which can be used in our case, that $J_{H}$ is a quasi-regular right ideal in 
$A_{H}$. Hence $A_{H} J_{H}=(A J) \otimes H$ is also a quasi-regular ideal in $A_{H}$ [2]. This implies by Theorem 4 that it is also a nil ideal, which by Remark 2 yields that it is also an LBI ideal. Clearly $A_{H} J_{H} \supset A J$ implies that $A J$ is also an LBI ideal. Q.E.D.

The proof of this fact for the LBD properties is more difficult and we shall need the following lemmas:

Lemma 7. Let $x \in A$ be an algebraic element and let $y A$ be an LBD right ideal in $A$; then $x+y$ is also algebraic.

We may assume that if $A$ is an algebra over $F$ then card $F>(A: F)$, since by extending the base field $F$ to a field of high cardinality the element $x$ remains algebraic and the ideal $y A$ remains an LBD ideal by Lemma 6 and if $x+y$ is algebraic over the extended field it is also algebraic over the base field. Next we assert that $N(x+y) \cup C(x+y)$ is the field $F$ with the possible exceptions of a finite number of elements. If this is shown then we may repeat the argument of Lemma 3 to show that card $C(x+y)=$ card $F$. That is, by Lemma 2 it follows that card $N(x+y) \leqq$ card $F$ and since the cardinal number of the union $N(x+y) \cup C(x+y)$ is equal to card $F$ then card $C(x+y)$ $=$ card $F$. The latter is by assumption greater than $(A: F)$, hence Theorem 3 implies that $x+y$ is algebraic. To prove our assertion we consider the element $-\lambda^{-1}(x+y)$ for $\lambda \notin N(x)$. Let $x_{\lambda}$ be the quasi-inverse of $-\lambda^{-1} x$ and let $q=-\lambda^{-1}(x+y)+x_{\lambda}-\lambda^{-1}(x+y) x_{\lambda}$ $=-\lambda^{-1} y\left(1+x_{\lambda}\right)$. If this element is quasi-regular then one readily verifies that $-\lambda^{-1}(x+y)$ is quasi-regular and, actually, a quasiinverse of $-\lambda^{-1}(x+y)$ will be the element $x_{\lambda}+q^{\prime}+x_{\lambda} q^{\prime}$ where $q^{\prime}$ is a quasi-inverse of $q$. Hence, in this case $\lambda \in C(x+y)$. If $q$ is not quasiregular, i.e. $-1 \notin S(q)$, then since $q \in y A, q$ is algebraic and by Lemma 1 it follows that since $-1 \in S(q),-1 \in N(q)$. Hence $(1+q) z$ $=0$ for some nonzero $z \in A .{ }^{2}$ Put $z^{\prime}=\left(1+x_{\lambda}\right) z$. Then one readily verifies that $\left(1-\lambda^{-1}(x+y)\right) z^{\prime}=\left(1-\lambda^{-1} y\left(1+x_{\lambda}\right)\right) z=(1+q) z=0$. Thus $\lambda \in N(x+y)$. The proof of the assertion follows now from the fact that since $x$ is algebraic, the set $N(x)$ contains only a finite number of elements.

This immediately implies:

COROLLARY 8. If $J$ is a right LBD ideal and $x$ is algebraic then $x+j$ is algebraic for every $j \in J$.

Lemma 8. If $J$ is a right $L B D$ ideal in $A$ then aJ is also a right $L B D$ ideal.

First we note that if an element $x a$ is algebraic and satisfies an equation $X^{n}+\alpha_{1} X^{n-1}+\cdots+\alpha_{n}=0$, then $a x$ satisfies the equation 
$X^{n+1}+\cdots+X \alpha_{n}=0$. This is obtained by multiplying the relation $(x a)^{n}+\cdots+\alpha_{n}=0$ on the right by $a$ and on the left by $x$. Hence the degree of the elements of the module $a j_{1}, \cdots, a j_{r}, j_{i} \in J$, is at most greater by 1 than the corresponding element in the module generated by $j_{1} a, \cdots, j_{r} a$, and the latter is of bounded degree since $j_{i} a \in J$.

We now turn back to the proof of Theorem 6. It remained to show that if $J_{H}$ is a right LBD ideal in $A_{H}$ then the ideal $A_{H} J_{H}$ is also an LBD ideal. Since $H$ was chosen to be nondenumerable, it suffices to show, in view of Remark 2, that this ideal is algebraic. Let $x=a_{1} j_{1}$ $+\cdots+a_{r} j_{r} \in A_{H} J_{H}$ where $a_{i} \in A$ and $j_{i} \in J_{H}$. We shall prove by induction on $r$ that the right-ideal $a_{1} J_{H}+\cdots+a_{r} J_{H}$ is an LBD algebra and this will imply that $x$ is algebraic. For $r=1$, this is included in Lemma 8. Assume now that $a_{1} J_{H}+\cdots+a_{r-1} J_{H}$ is a right LBD ideal. Let $a_{r} y \in a_{r} J_{H}$. Then it is algebraic. Thus it follows by Corollary 8 that $a_{r} y+z$ is algebraic for every $z \in a_{1} J_{H}+\cdots+a_{r-1} J_{H}$. This means that $a_{1} J_{H}+\cdots+a_{r-1} J_{H}+a_{r} J_{H}$ is algebraic and therefore, by Remark 2, it is also a right LBD ideal. This completes the proof of Theorem 6.

It follows now by the results of II that we can speak of the LBD radical and the LBI radical of every algebra $A$. We shall denote them by $\operatorname{LBD}(A)$ and $\operatorname{LBI}(A)$ respectively. Theorem 6 shows that these two radicals satisfy the same properties as the semi-nilpotent radical [8] (see also III, Theorem 2) which we shall denote by $\operatorname{LN}(A)$ and the locally finite kernel ([10] and also example B of III, §3) which is denoted by $\operatorname{LF}(A)$. Clearly every semi-nilpotent algebra is also an LBI algebra and the latter is a nil algebra. Similarly, every locally finite ideal is an LBD algebra, and the latter is an algebraic algebra. Hence:

TheoRem 7. $\Re(A) \supseteq L B I(A) \supseteq L N(A) ; \mathfrak{A}(A) \supseteq L B D(A) \supseteq L F(A)$, where $\Re(A)$ and $\mathfrak{X}(A)$ denote respectively the maximal nil and algebraic ideals of $A$.

Still remaining unsettled is the old problem of Kurosh [6] which can be stated as the problem of substituting the equality sign in the two groups of the radicals of this theorem. It follows only by Remark 2 that:

CoRollary 9. If $A$ is an algebra over a nondenumerable field, then $\Re(A)=L B I(A)$ and $\mathfrak{A}(A)=L B D(A)$.

Theorem 6 implies also by the results of II, similar to the results of III, Theorem 2.1 and its corollary, that: 
THEOREM 8. $L B D(Q)=L B D(A) \cap Q, L B I(Q)=L B I(A) \cap Q$ for every ideal $Q \subseteq A$, and $L B D\left(A_{n}\right)=[L B D(A)]_{n}, L B I\left(A_{n}\right)=[L B I(A)]_{n}$ where $A_{n}$ denotes the complete matrix ring of order $n^{2}$ over $A$.

In particular if $A$ is an algebraic algebra over a nondenumerable field $F$ then $\operatorname{LBD}(A)=A$ by Corollary 9 . Hence the last theorem, together with Lemma 6 and Remark 2, yields a solution of the problems proposed by Jacobson [2] for algebras over nondenumerable fields:

THEOREM 9. If $A$ is an algebraic algebra over a nondenumerable field $F$ then every extension algebra $A_{H}$ and matrix algebra $A_{n}$ are algebraic algebras.

By Remark 2 and by II, Theorem 3.2 it follows:

COROLLARY 10. The union of right algebraic (nil) ideals of an algebra over a nondenumerable field is a right algebraic (nil) ideal.

REMARK 3. By Theorem 6 and II, Theorem 3.2, it follows that the union of right $\mathrm{LBD}(\mathrm{LBI})$ ideals of algebras over infinite fields is also a right $\mathrm{LBD}(\mathrm{LBI})$ ideal.

Theorem 6 and Remark 2 together with the results of II, $\$ 3$ yield the solution of the problem of Koethe [6] for algebras over nondenumerable fields:

THEOREM 10. If $A$ is an algebra over a nondenumerable field then the maximal nil ideal $\Re(A)$ of $A$ contains all one-sided right ideals of $A$.

Furthermore, by Theorem 8 it follows that:

COROLLARY 11. If $A$ is a nil algebra over a nondenumerable field then the matrix ring $A_{n}$ over $A$ is also a nil algebra.

\section{BIBLIOGRAPHY}

1. S. A. Amitsur, A general theory of radicals, II, Amer. J. Math. vol. 76 (1954) pp. 100-125; part III, ibid. vol. 76 (1954) pp. 126-136.

2. N. Jacobson, The radical and semi-simplicity for arbitrary rings, Amer. J. Math. vol. 67 (1945) pp. 300-320.

3. - Structure theory for algebraic algebras of bounded degree, Ann. of Math. vol. 46 (1945) pp. 695-707.

4. I. Kaplansky, Rings with a polynomial identity, Bull. Amer. Math. Soc. vol. 54 (1948) pp. 575-580.

5. - Topological representation of algebras, II, Trans. Amer. Math. Soc. vol. 68 (1950) pp. 62-75.

6. G. Koethe, Die Structur der Ringe deren Restklassenring dem Radical vollständig ist, Math. Zeit. vol. 32 (1930) pp. 161-186. 
7. A. Kurosh, Ringtheoretische Probleme die mit dem Burnsideschen Problem über periodische Gruppen in Zussammenhang stehen, Bull. Acad. Sci. URSS, Sér. Math. vol. 5 (1941) pp. 233-240.

8. J. Levitzki, On the radical of a general ring, Bull. Amer. Math. Soc. vol. 49 (1943) pp. 462-466.

9. - On three problems concerning nil rings, Bull. Amer. Math. Soc. vol. 49 (1943) pp. 913-919.

10. - On the structure of algebraic algebras and related rings, Trans. Amer. Math. Soc. vol. 74 (1953) pp. 384-409.

HEBREW UNIVERSITY

\section{ON THE SHORTEST SPANNING SUBTREE OF A GRAPH AND THE TRAVELING SALESMAN PROBLEM}

JOSEPH B. KRUSKAL, JR.

Several years ago a typewritten translation (of obscure origin) of [1] raised some interest. This paper is devoted to the following theorem: If a (finite) connected graph has a positive real number attached to each edge (the length of the edge), and if these lengths are all distinct, then among the spanning ${ }^{1}$ trees (German: Gerüst) of the graph there is only one, the sum of whose edges is a minimum; that is, the shortest spanning tree of the graph is unique. (Actually in [1] this theorem is stated and proved in terms of the "matrix of lengths" of the graph, that is, the matrix $\left\|a_{i j}\right\|$ where $a_{i j}$ is the length of the edge connecting vertices $i$ and $j$. Of course, it is assumed that $a_{i j}=a_{j i}$ and that $a_{i i}=0$ for all $i$ and $j$.)

The proof in [1] is based on a not unreasonable method of constructing a spanning subtree of minimum length. It is in this construction that the interest largely lies, for it is a solution to a problem (Problem 1 below) which on the surface is closely related to one version (Problem 2 below) of the well-known traveling salesman problem.

Problem 1. Give a practical method for constructing a spanning subtree of minimum length.

Problem 2. Give a practical method for constructing an unbranched spanning subtree of minimum length.

The construction given in [1] is unnecessarily elaborate. In the present paper I give several simpler constructions which solve Problem 1, and I show how one of these constructions may be used to prove the theorem of [1]. Probably it is true that any construction

Received by the editors April 11, 1955.

${ }^{1}$ A subgraph spans a graph if it contains all the vertices of the graph. 